\title{
INVESTIGATION OF THE SPONTANEOUS SPIN-FLIP PHASE TRANSITION IN TERBIUM-YTTRIUM IRON-GARNET BY THE MAGNETOOPTIC METHOD
}

\author{
D.R. DJURAEV, L.N. NIYAZOV, K.S. SAIDOV, B.YU. SOKOLOV
}

PACS 75.30.Kz, 05.30.Rt (C) 2012

\author{
Bukhara State University \\ (Bukhara 205018, Uzbekistan; e-mail: djuraev2002@mail.ru)
}

\begin{abstract}
The domain structure transformation and the technical magnetization of a $\mathrm{Tb}_{0.26} \mathrm{Y}_{2.74} \mathrm{Fe}_{5} \mathrm{O}_{12}$ single crystal have been studied by the magnetooptic method in the temperature region of a spontaneous spin-flip phase transition (SFPT). It has been found that the SFPT occurs in a finite temperature interval, where the low- and high-temperature magnetic phase domains coexist. We have observed the anomalies of the temperature dependences of the coercive force and the magnetooptic susceptibility of the crystal related to a transformation of its domain structure under the spin-flip. The experimental results obtained are interpreted within the framework of the SFPT theory for a cubic crystal. It has been demonstrated that the existing theory describes consistently the evolution of the $\mathrm{Tb}_{0.26} \mathrm{Y}_{2.74} \mathrm{Fe}_{5} \mathrm{O}_{12}$ garnet domain structure under the spontaneous reorientation of the easy magnetization axis.
\end{abstract}

\section{Introduction}

Rare-earth ferrite-garnets are the traditional object for studies in the physics of magnetic phase transitions of the order - disorder type [1]. Recently, the interest in such investigations is stimulated by the perspective of fabrication of materials for the element bases of newgeneration devices of spintronics on the basis of rareearth ferrite-garnets [2]. It is known that, as the temperature decreases from room one down to $T=T_{\text {sf }}$ ( $T_{\mathrm{sf}}$ is the spin-flop temperature) in rare-earth ferritegarnets of the system $\mathrm{Tb}_{x} \mathrm{Y}_{3-x} \mathrm{Fe}_{5} \mathrm{O}_{12}$, the easy magnetization axis reorients from crystallographic directions of the type $\langle 111\rangle$ to directions of the type $\langle 100\rangle[3$, 4]. The theory of this spontaneous spin-flip phase transition developed in [3] describes well the available results of experimental studies of magnetic properties of the system $\mathrm{Tb}_{x} \mathrm{Y}_{3-x} \mathrm{Fe}_{5} \mathrm{O}_{12}$ near $T_{\mathrm{sf}}$ that are obtained at a sufficiently strong magnetic field $H$ ensuring the single-domain state of the crystal $[3,4]$. At the same time, it is obvious that many magnetic characteristics of rare-earth ferrite-garnets (initial susceptibility, coercive force, rate of remagnetization, and so on), which are actual for practical applications, are determined to a significant extent by the realized domain structure [5]. The theory of a rearrangement of the domain structure of a cubic crystal under SFPT was proposed in $[3,4]$ more that three decades ago. But, to the best of our knowledge, no direct experimental studies of this process running in rare-earth ferrite-garnets have been executed. In this connection, we carried out the visual observations of the evolution of the domain structure and studied the process of magnetization of garnet $\mathrm{Tb}_{0.26} \mathrm{Y}_{2.74} \mathrm{Fe}_{5} \mathrm{O}_{12}$ in the temperature region of its spin-flop. The appropriate results are given in what follows.

\section{Specimens and the Experimental Procedure}

In the studies, we used a single crystal of garnet $\mathrm{Tb}_{0.26} \mathrm{Y}_{2.74} \mathrm{Fe}_{5} \mathrm{O}_{12}$ grown by the method of spontaneous crystallization from a melt. From it, we cut thin planeparallel plates so that their developed surfaces were in parallel to the crystallographic plane (110). After a mechanical polishing, the surfaces of specimens undergo (to remove the near-surface layer disturbed by a mechanical processing) the chemical etching with orthophosphoric acid. The specimens prepared for experiments have a thickness of $\sim 80 \mu \mathrm{m}$ and the transverse sizes of $\sim 2 \times 2 \mathrm{~mm}^{2}$.

The results of the theoretical analysis of a magnetic structure of rare-earth ferrite-garnets of the system $\mathrm{Tb}_{x} \mathrm{Y}_{3-x} \mathrm{Fe}_{5} \mathrm{O}_{12}$ imply that the stable directions of a spontaneous magnetic moment $\mathbf{M}$ in a crystal are the directions $\langle 111\rangle$ in the region of temperatures of the order of room one [3, 4]. Hence, at a chosen crystallographic orientation of a specimen, there are four directions lying in the plane of the specimen, and four directions at angles of $\pm 55^{\circ}$ relative to the plane of the specimen, along which $\mathbf{M}$ can lie at room temperature. However, it follows from the general physical ideas that the domain structure consisting of domains magnetized in the plane of the plate has the highest energy gain 

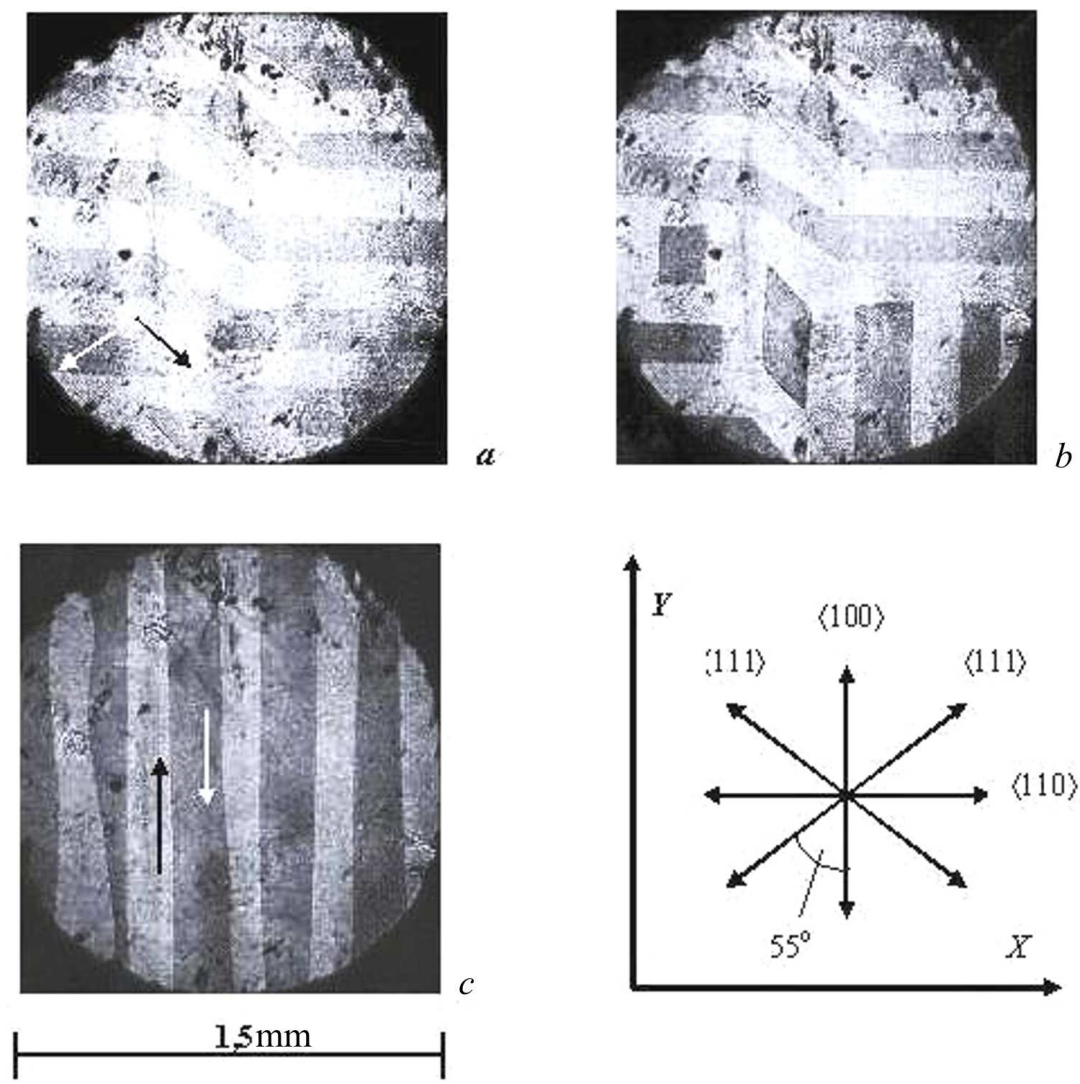

Fig. 1. Images of the domain structure of the specimen of garnet $\mathrm{Tb}_{0.26} \mathrm{Y}_{2.74} \mathrm{Fe}_{5} \mathrm{O}_{12}$ obtained in the zero magnetic field at various temperatures: $a-290, b-129, c-120 \mathrm{~K}$. The specimen is turned around the axes $X$ and $Y$ relative to the focal plane of a microscope by angles of $\approx 15^{\circ}$. Arrows indicate the directions of a spontaneous magnetic moment in adjacent domains. In the insert: the orientation of principal crystallographic directions in the plane of the specimen and the axes of the laboratory coordinate system (the axis $Z$ is the direction of light falling onto the crystal)

due to the significantly less demagnetizing factor in the plane of the thin plate relative to that in the perpendicular direction. In view of this, we may expect that the domain structure of the specimens under study in the region of high temperatures consists of domains, in which the vector $\mathbf{M}$ lies in their plane, with $110^{\circ}$ and $70^{\circ}$ domain walls of the Néel type (the orientation of the axes of the laboratory coordinate system and the directions of main crystallographic axes in the plane of a specimen are shown in Fig. 1). Nevertheless, the observations showed that such domain structure exists at $T=290 \mathrm{~K}$ not in all cases: a part of specimens possesses a blind irregular domain structure which contains sections, in which the vector $\mathbf{M}$ lies in the plane of the specimen, as well as sections, in which the vector $\mathbf{M}$ is perpendicular (or almost perpendicular) to its plane. It is known that an irregular domain structure indicates the presence of inhomogeneous mechanical stresses (growth ones and/or stresses induced by a mechanical processing) in a crystal [6]. Therefore, we chose, for experiments, the specimens free from mechanical stresses and possessing a domain configuration that is the most "proper" from the theoretical viewpoint.

The visualization of the domain structure was realized with the use of the standard magnetooptic method: the domains were observed with the help of a polarizing microscope in the transmission mode; the contrast of the obtained images of the domain structure was mainly determined by different signs of the Faraday rotation in adjacent domains. Since the vector $\mathbf{M}$ in the chosen specimens lies in their planes, we oriented the specimens to visualize the domain structure so that the incident angle was $\approx 15^{\circ}$ (the specimen was rotated around the axis normal to the average direction of magnetization) ensuring the presence of the component of $\mathbf{M}$ along the direction of light propagating in the crystal, which de- 
termined the Faraday effect. ${ }^{1}$ In experiments, we used a long-focus objective; the total magnification of a microscope was $40^{\mathrm{x}}$. The obtained images of the domain structure were fixed by a digital photochamber connected with a computer.

In addition to the visual observation of the domain structure of $\mathrm{Tb}_{0.26} \mathrm{Y}_{2.74} \mathrm{Fe}_{5} \mathrm{O}_{12}$ and its evolution at a change of the temperature, we studied the temperature dependences of two characteristics of the crystal directly related to its domain structure: the field dependences of the Faraday effect $\alpha(H)$ ( $\alpha$-angle of the Faraday rotation) and the magnetooptic susceptibility $\partial \alpha / \partial H$. The measurements of the quantities $\alpha$ and $\partial \alpha / \partial H$ were carried out at the same orientation of the specimen, as that in visual observations of its domain structure, by the ordinary intensity-involving procedure (the angle between the axes of a polarizer and an analyzer was $45^{\circ}$ ) in an alternative magnetic field varying by the harmonic law with a frequency of $25 \mathrm{~Hz}$. In experiments, we measured the ratio $\Delta I / I$, where $\Delta I$ and $I$ are, respectively, the varying and constant components of the light flow passing the polarizer-specimen-analyzer system. For the angles $\alpha \leq 1^{\circ}$ measured in experiments, the value of $\Delta I$ depends linearly with good accuracy on $\alpha$ [7]. Since the angle $\alpha$ is proportional to the magnetization of a specimen, it is obvious that the dependence $\alpha(H) \propto \Delta I(H) / I$ reflects the course of a technical magnetization curve, and the dependence $\partial \alpha / \partial H(T) \propto 1 / I \partial \Delta I / \partial H(T)$ corresponds to the temperature dependence of the initial magnetic susceptibility. For the magnetization of a specimen, we used a pair of Helmholtz coils, whose axis lay in the plane of a specimen. In the studies of the dependence $\alpha(H)$, the magnetic field amplitude was 40 Oe, and the value of $\partial \alpha / \partial H(T)$ was 0.2 Oe (in both cases, the vector $\mathbf{H}$ was oriented in parallel to the surface of a specimen).

All studies were carried out in the temperature interval $85 \div 290 \mathrm{~K}$, and the rate of variation of the temperature at the heating (cooling) of a specimen was $\approx 0.3 \mathrm{~K} / \mathrm{s}$.

\section{Experimental Results and Their Discussion}

According to the theory of SFPT developed in $[3,4]$, the orientation of the easy magnetization axis in garnets $\mathrm{Tb}_{x} \mathrm{Y}_{3-x} \mathrm{Fe}_{5} \mathrm{O}_{12}$ is determined by the ratio of the values

\footnotetext{
1 At such an orientation, the plane of a specimen deviates from the focal plane of a microscope. Therefore, the slope angle of the specimen was limited by the optical definition depth of a microscope.
}

of the first, $K_{1}$, and second, $K_{2}$, constants of cubic magnetic anisotropy, which have different temperature dependences. At $K_{1} \leq-K_{2} / 3$ (the region of temperatures close to room one), the orientation of $\mathbf{M}$ along axes $\langle 111\rangle$ (magnetic phase $\Phi_{111}$ ) is stable, whereas, at $K_{1} \geq 0$, the orientation along axes $\langle 100\rangle$ (magnetic phase $\Phi_{100}$ ) is stable. As is seen from the presented relations, there exists the region of values of the constants $K_{1}$ and $K_{2}$ (in some interval of temperatures), in which the phases $\Phi_{111}$ and $\Phi_{100}$ coexist (the sign of equality in these relations corresponds to the points, where the phase with a certain orientation of $\mathbf{M}$ loses the stability).

As the temperature decreases below room one, the constant $K_{1}$ changes the sign, and, under the condition

$9 K_{1}+K_{2}=0$

the easy magnetization axis reorients itself by jump from the direction $\langle 111\rangle$ to $\langle 100\rangle$. According to the calculations performed in $[1,4]$ on the basis of experimentally obtained dependences $K_{1}(T)$ and $K_{2}(T)$, condition (1) for $\mathrm{Tb}_{0.26} \mathrm{Y}_{2.74} \mathrm{Fe}_{5} \mathrm{O}_{12}$ is satisfied at the temperature $T_{\text {sf }} \approx 130 \mathrm{~K}$.

In Fig. 1, $a$, we present the pattern of the domain structure of one of the selected specimens, which was observed at $T=290 \mathrm{~K}$ in the zero magnetic field. The arrows show the direction of the vector $\mathbf{M}$ in domains. This direction was determined by the reaction of the domain structure to an external magnetic field: under the magnetization of a specimen in a plane, the areas of domains, in which the direction of the vector $\mathbf{M}$ takes an acute angle with the vector $\mathbf{H}$, increased at the expense of domains with less favorable orientations of $\mathbf{M}$. In this case at the rotations of a specimen around the axis $Y$ by an angle of $\pm 15^{\circ}$ (it should be so at $\mathbf{M}$ lying in the plane of a specimen), the dark domains became light ones, and the light domains became dark ones at the invariable contrast between adjacent domains.

On the basis of Fig. 1,a, we may conclude that a domain configuration completely corresponding to the theoretical ideas is realized in the specimen. Hence, the domain wall of the observed domain structure includes the sections, where the vector $\mathbf{M}$ is parallel to the easy magnetization axis of the phase $\Phi_{100}$. According to the model accepted in $[3,4]$, those sections of the domain wall are "nuclei" of the phase $\Phi_{100}$. Moreover, at a reorientation of the easy axis direction, the domains of a new phase "grow" from the domain wall of the old phase. In other words, a domain structure in the region of SFPT $\Phi_{111} \rightarrow \Phi_{100}$ always exists in the crystal. However, another scenario of a rearrangement of the domain structure is possible. If we consider that the domain 


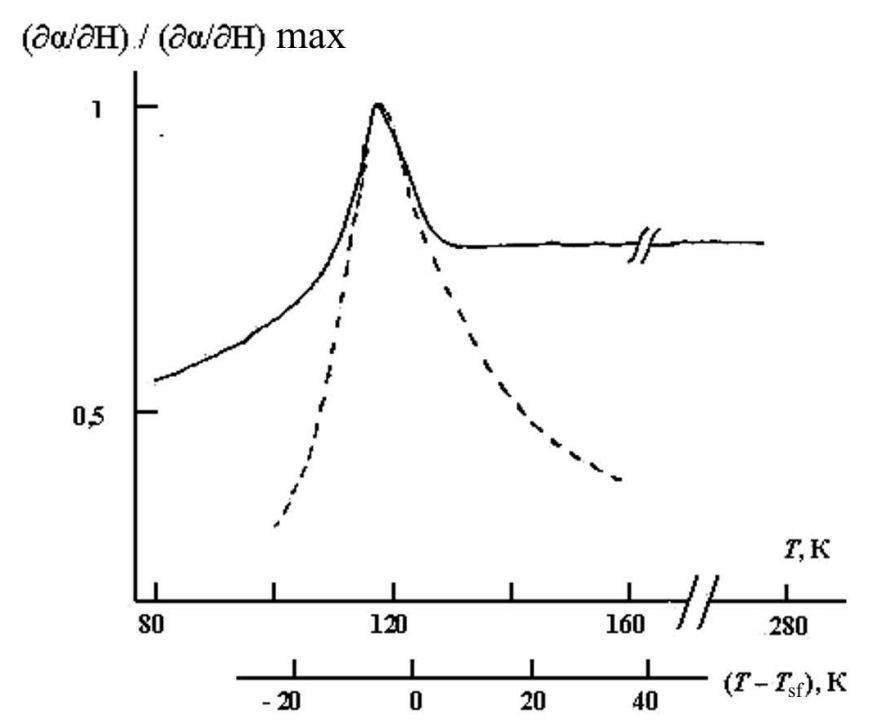

Fig. 2. Temperature dependence of the magnetooptic susceptibility of the specimen normalized to its maximum value. Solid fine - experiment $(\mathbf{H} \|\langle 111\rangle)$, dotted line (lower temperature scale) calculation by formula (3). The specimen is oriented in the focal plane of a microscope in the same manner as in the study of the domain structure

wall width is $\delta \propto\left(A / K_{\text {eff }}\right)^{1 / 2}$, where $A$ is the exchange constant, and $K_{\text {eff }}$ is the constant of effective magnetic anisotropy [8], then, under condition (1), $K_{\text {eff }} \rightarrow 0$, and $\delta \rightarrow \infty$. In this case, the rearrangement of the domain structure must occur due to the growth of the domain wall width. In other words, the domains of the phase $\Phi_{111}$ must disappear in some temperature interval near $T_{\mathrm{sf}}$, and only then the domains of the phase $\Phi_{100}$ will appear.

The direct visual observations indicate that, though the domain structure of the specimen under study is changed considerably in the region of low temperatures, the domains exist in the whole interval $85 \leq T \leq 290 \mathrm{~K}$. At the cooling below $T=290 \mathrm{~K}$, the domain structure is not practically changed down to $T \approx 132 \mathrm{~K}$. Below this temperature in some region of the specimen, the domains of the low-temperature magnetic phase appear by jump with the orientation of the vector $\mathbf{M}$ along the axis $\langle 100\rangle$ (Fig. 1,b) instead of the domains of the high-temperature phase. The former smoothly "intergrow" on the whole area of the specimen, as the temperature decreases. The rearrangement of the domain structure of the specimen occurs in the temperature interval $\Delta T \approx 12 \mathrm{~K}$ (in this temperature interval, we see the coexistence of domains of the old and new phases). After this, the image of the specimen contains only the domains of the phase $\Phi_{100}$ (Fig. 1,c). ${ }^{2}$ In this case, the period of the domain structure in the interval $\Delta T$ (like in the region $T>132 \mathrm{~K}$ ) does not depend practically on $T$ and only decreases slightly (approximately by 15 ), as the temperature decreases down to $T=85 \mathrm{~K}$. This seems unexpected from the viewpoint of the accepted theory of a domain structure of the Cotton type. Indeed, according to [8], the period of a Cotton domain structure is $D \propto\left(A / K_{\text {eff }}\right)^{1 / 2}$. If we use the data on the temperature dependence of the constant $K_{\text {eff }}$ (see Figs. 2 and 3), this yields a sharper decrease in the period of the domain structure in the low-temperature region as compared with the results of visual observations.

As the temperature increases from $T=85 \mathrm{~K}$, the rearrangement of the domain structure of the specimen occurs in the inverse order: the domains of the hightemperature phase gradually occupy the place of domains of the low-temperature phase in the same (in the limits of errors of the experiment, $\sim 1 \mathrm{~K}$ ) interval $\Delta T$. However, the temperatures of the onset and the end of the rearrangement of the domain structure were shifted upward by approximately $5 \mathrm{~K}$ relative to the values of relevant temperatures obtained at the cooling of the specimen. At the same time, according to the theory developed in $[3,4]$, the rearrangement of the domain structure in a many-domain crystal in the region of the transition $\Phi_{111} \rightarrow \Phi_{100}$ must run invertibly.

Except for the temperature hysteresis, the abovedescribed behavior of the domain structure agrees qualitatively with the consequences of the theory of SFPT which are mentioned at the beginning of this section. Moreover, the temperature region and the interval $\Delta T$, where we observe a remarkable change of the domain structure, are in satisfactory agreement with the temperature intervals calculated in [4], where the phases $\Phi_{111}$ and $\Phi_{100}$ coexist.

In Fig. 2, we present the temperature dependence of the magnetooptic susceptibility of the specimen under study, which is normalized to its maximum value $(\delta \alpha / \delta H)_{\max }$. This dependence was obtained at the orientation of $\mathbf{H}$ in the specimen plane along the direction $\langle 111\rangle$ (the experimentally measured value $(\delta \alpha / \delta H)_{\max }=\delta \alpha / \delta H(T=125 \mathrm{~K})=0.025$ degree $\left./ \mathrm{Oe}\right)$. It is seen that, at high $T$, the ratio $(\delta \alpha / \delta H) /(\delta \alpha / \delta H)_{\max }$ does not depend practically on the temperature down to $T \approx 132 \mathrm{~K}$ and then start to increase, by at-

${ }^{2}$ It is obvious that, at the temperatures of the onset and the end of a rearrangement of the domain structure of the specimen, the appropriate magnetic phases lose the stability. 
taining the maximum at $T \approx 125 \mathrm{~K}$. Then the ratio $(\delta \alpha / \delta H) /(\delta \alpha / \delta H)_{\max }$ sharply decreases and is equal to $\sim 75$ at $T=85 \mathrm{~K}$ and 1 at room temperature. We note that the anomaly of the dependence $\delta \alpha / \delta H(T)$ near $T=125 \mathrm{~K}$ is more pronounced as compared with an analogous specific feature of the high-frequency magnetic susceptibility of garnet $\mathrm{Tb}_{0.26} \mathrm{Y}_{2.74} \mathrm{Fe}_{5} \mathrm{O}_{12}$, which was observed on the bulk specimen in [3]. The fall of the curve $\delta \alpha / \delta H(T)$ at $T<125 \mathrm{~K}$ is less sharp.

In Fig. 3, we show the temperature dependence of the coercive force of the same specimen, which is normalized to its minimum value $H_{\mathrm{c}(\min )}=H_{\mathrm{c}}(T=125 \mathrm{~K})=4.2$ Oe. There, we also present the field dependences of the Faraday effect measured at $\mathbf{H} \|\langle 111\rangle$ in the temperature region $T \leq 160 \mathrm{~K}$ (above $T=160 \mathrm{~K}$, the shape of the curve $\alpha(H)$ is independent of $T$ in the limits of experimental errors). It follows from the plots given in Fig. 3 that, in particular, the slope of the curve $\alpha(H)$ at $T=125 \mathrm{~K}$ relative to the abscissa axis and the coercive force $H_{\mathrm{c}}$ are minimum. ${ }^{3}$

It is remarkable that the performed studies indicate the absence (as distinct from the results of visual observations of the rearrangement of the domain structure) of the temperature hysteresis for the dependences $\delta \alpha / \delta H(T)$ and $H_{\mathrm{c}}(T)$. We also note that the temperatures of the maximum on the dependence $\delta \alpha / \delta H(T)$ and the minimum on the dependence $H_{\mathrm{c}}(T)$ are identical. To explain the mentioned difference between the behaviors of the domain structure and the quantities $H_{\mathrm{c}}$ and $\delta \alpha / \delta H$, we need to take into account that, at the spontaneous reorientation of the easy axis, the domain wall is significantly shifted. This displacement in a real crystal is accompanied by the effect of pinning on dislocations and other bulk defects of the crystal lattice. It is natural that some energy should be spent in order to overcome the pinning of a domain wall and to destroy the related metastable domain configurations. Near SFPT at $H=0$, it is the energy of a magnetocrystal anisotropy, which is additional relative to the energy of the ideal crystal. Therefore, the evolution of the domain structure of a specimen under study is running, by revealing the temperature hysteresis. We may assume that the variable magnetic field with a sufficient strength breaks away the domain walls from the pinning centers, by destroying the metastable domain configurations, which leads to the absence of the temperature hysteresis for the de-

\footnotetext{
3 Some asymmetry of the shape of the observed hysteresis loops is related, probably, to the contribution to the intensity of light falling onto an analyzer that is even with respect to the direction of the vector $\mathbf{M}$ and is caused by the magnetic linear birefringence of the crystal.
}

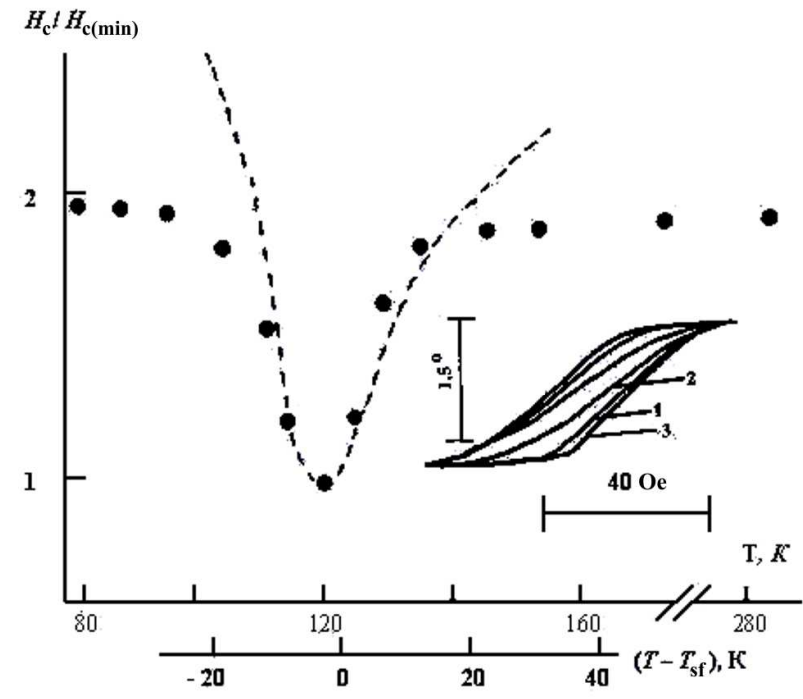

Fig. 3. Temperature dependence of the coercive force of the specimen normalized to its minimum value. Points - experiment, dotted line (lower temperature scale) - calculation by formula (2). In the insert: the oscillograms of loops of the magnetic hysteresis of the Faraday effect observed at various temperatures: $1-160$, 2 $125,3-85 \mathrm{~K}$. The orientation of the specimen and the direction of the magnetic field are the same as those in the studies of the dependence $\delta \alpha / \delta H(T)$

pendences $\delta \alpha / \delta H(T)$ and $H_{\mathrm{c}}(T)$. As was shown by the visual observations carried out on the same specimen in a variable magnetic field with an amplitude of 0.2 Oe $(\mathbf{H} \|\langle 111\rangle)$, the hysteresis of the temperatures of the onset and the end of the rearrangement of its domain structure decreases, indeed, to the level of the measurement error for $T$ related to the inertia of heating-cooling of a specimen. Probably, the above-mentioned significant decrease of the period of the domain structure of the specimen under study with decrease in the temperature is also related to the pinning of domain walls.

As is known, the shape of the curve of a technical magnetization of a many-domain crystal is determined in the general case by two processes: a displacement of domain walls and the rotation of $\mathbf{M}$. In the spin-flop region, where the domain structure becomes unstable and the magnetic anisotropy decreases, an external field $H$ must cause the most intense displacement of domain walls and the most rapid turn of the vector $\mathbf{M}$ to the side of $\mathbf{H}$. It is obvious that the magnetic properties of a crystal near $T_{\text {sf }}$ will depend on that which of these processes is dominant. This is of importance from the viewpoint of a perspective of technical applications of the material and for the choice of an adequate theoretical model of SFPT. 
Since, at the present time, we have no complete theory of the displacement of domain walls under the action of $H$, the quantitative estimates of the influence of this process on the magnetic characteristics of a crystal are difficult (in particular, to make such estimates, we need the data on such parameters hard to be controlled as the pinning strength of domain walls, the bulk density of pinning centers, etc). However, it is seen from Fig. 3 that the hysteresis loop in the temperature region of SFPT becomes narrower (the coercive force $H_{\mathrm{c}}$ decreases), and the dependence $\alpha(H)$ approaches a linear one far from the saturation. Such hysteresisless magnetization curve is characteristic of the rotation of $\mathbf{M}$ [6]. Therefore, we may conclude that the magnetic properties of the specimen under study near the spin-flip point are mainly determined by a turn of the vector $\mathbf{M}$ to the magnetization direction. In this case, (see, e.g., [6]) the formula for the coercive force looks as

$H_{\mathrm{c}} \propto \frac{K_{\mathrm{eff}}}{M}$,

and the magnetooptic susceptibility is given by

$\frac{\delta \alpha}{\delta H} \propto \frac{M^{2}}{K_{\mathrm{eff}}}$,

where $K_{\text {eff }}=\left|K_{2}\right| / 3-K_{1}$ for $T>T_{\text {sf }}$, and $K_{\text {eff }}=K_{1}$ for $T<T_{\text {sf }}[3]$.

The dependences $\delta \alpha / \delta H(T) / \delta \alpha /(\delta H)_{\max }$ and $H_{\mathrm{c}}(T) / H_{\mathrm{c}(\min )}$ calculated by formulas (3) and (2) with the use of the temperature dependences of the constants $K_{1}$ and $K_{2}$ presented in $[1,4]^{4}$ are shown in Figs. 2 and 3 . As is seen from the comparison of the calculated and experimental curves, formulas (2) and (3) describe satisfactorily the temperature dependences of the coercive force and the magnetooptic susceptibility of the specimen under study in the temperature region of a rearrangement of its domain structure.

Relations (2) and (3) imply that, at the SFPT point, where $K_{\text {eff }}$ is minimum, the quantities $\delta \alpha / \delta H$ and $K_{\mathrm{c}}$ must pass through the maximum and the minimum, respectively. This is observed experimentally at $T=125 \mathrm{~K}$ (see Figs. 2 and 3), which means that $T=125 \mathrm{~K}$ is the temperature of the phase transition $\Phi_{111} \leftrightarrow \Phi_{100}$ in the specimen. It turns out to be by $\sim 5 \mathrm{~K}$ less than that calculated by formula

\footnotetext{
${ }^{4}$ In this case, we consider that $\mathrm{M}=$ const in the interval of temperatures used in the plots.
}

(1) and by $\sim 10 \mathrm{~K}$ less than the values of $T_{\mathrm{sf}}$ determined in $[3,4]$ by the results of experimental studies of the high-frequency magnetic susceptibility and the nuclear magnetic resonance in a bulk crystal of garnet $\mathrm{Tb}_{0.26} \mathrm{Y}_{2.74} \mathrm{Fe}_{5} \mathrm{O}_{12}$.

It is obvious that, in real crystals of $\mathrm{Tb}_{0.26} \mathrm{Y}_{2.74} \mathrm{Fe}_{5} \mathrm{O}_{12}$ with sufficiently large size, some mechanical stresses (related, e.g., to an inhomogeneity of their chemical composition, which is characteristic of many-component rareearth ferrite-garnets [5]) are always present. Therefore, we can assert that the behavior of the magnetic properties of bulk crystals (which were studied in $[1,3,4])$ in the region of SFPT is determined by not only the dependences $K_{1}(T)$ and $K_{2}(T)$, but also by the temperature dependence of the anisotropy constant caused by mechanical stresses in the crystal lattice $^{5}$ Probably, this is the reason for the difference of our value of the transition temperature $\Phi_{111} \leftrightarrow$ $\Phi_{100}$ in $\mathrm{Tb}_{0.26} \mathrm{Y}_{2.74} \mathrm{Fe}_{5} \mathrm{O}_{12}$ from that determined in $[3,4]$.

\section{Conclusion}

The performed studies show that the available theory of SFPT describes adequately the rearrangement of the domain structure arising in a plane-parallel plate of garnet $\mathrm{Tb}_{0.26} \mathrm{Y}_{2.74} \mathrm{Fe}_{5} \mathrm{O}_{12}$ in the crystallographic plane (110) at the phase transition $\Phi_{111} \leftrightarrow \Phi_{100}$. The value of temperature interval, in which a considerable change of the domain structure occurs, is in satisfactory agreement with the temperature region of coexistence of the phases $\Phi_{111}$ and $\Phi_{100}$ calculated in [4] on the basis of a phase diagram describing the magnetic state of this garnet. The evolution of the domain structure in the process of spontaneous spin-flip reorientation runs with a temperature hysteresis related to the pinning of domain walls on defects of the crystal lattice, which were not considered in the theory of SFPT developed in $[3,4]$. The observed "spreading" of the transition $\Phi_{111} \leftrightarrow \Phi_{100}$ is caused by the coexistence of domains of the low- and high-temperature magnetic phases in some interval of temperatures. The studied dependences $H_{\mathrm{c}}(T)$ and $c \delta \alpha / \delta H(T)$ manifest the anomalies in the temperature region of a spontaneous reorientation of the axis of easy magnetization. The character of the anomalies is in qualitative agreement with the predic-

\footnotetext{
${ }^{5}$ As was indicated in the previous section, the irregular domain structure observed in a part of specimens indicates the presence of mechanical stresses in them, which confirms the given conclusion.
} 
tion of the available theory of SFPT in rare-earth ferritegarnets.

The present work is partially supported by the grant FA-F2-F104+F105 of the Academy of Sciences of the Republic of Uzbekistan.

1. 1. K.P. Belov, A.K. Zvezdin, A.M. Kadomtseva, and R.Z. Levitin, Orientational Transitions in Rare Earth Magnetics (Nauka, Moscow, 1979) (in Russian).

2. A.K. Zvezdin and A.P. Pyatakov, Usp. Fiz. Nauk 179, 897 (2009).

3. K.P. Belov, A.K. Zvezdin, R.Z. Levitin, A.S. Markosyan, B.V. Mill', A.A. Mukhin, and A.P. Perov, Zh. Eksp. Teor. Fiz. 68, 1189 (1975).

4. V.A. Borodin, V.D. Doroshev, V.A. Klochan, N.M. Kovtun, R.Z. Levitin, and A.S. Markosyan, Zh. Eksp. Teor. Fiz. 70, 1363 (1976).

5. V.V. Randoshkin and A.Ya. Chervonenkis, Applied Magnetooptics (Energoatomizdat, Moscow, 1990) (in Russian).

6. S. Chikazumi, Physics of Ferromagnetism (Oxford Univ. Press, Oxford, 1997).

7. M.M. Chervinskii, S.F. Glagolev, and V.B. Arkhangel'skii, Methods and Means of Measurements of Magnetic Characteristics of Films (Energoatomizdat, Leningrad, 1990) (in Russian).
8. Yu.V. Gulyaev, P.E. Zilberman, R.J. Elliot, and E.M. Epshtein, Fiz. Tverd. Tela 44, 1064 (2002).

Received 22.04.11.

Translated from Russian by V.V. Kukhtin

ДОСЛІДЖЕННЯ СПОНТАННОГО ОРЕНТАЦІЙНОГО ФАЗОВОГО ПЕРЕХОДУ У ТЕРБІЙ-ІТРІЕВОМУ ФЕРИТІ-ГРАНАТІ МАГНІТООПТИЧНИМ МЕТОДОМ

Д.Р. Дюжураєв, Л.Н. Ніязов, К.С. Саідов, Б.Ю. Соколов

$\mathrm{P}$ е $з$ ю м е

Магнітооптичним методом досліджено перебудову доменної структури і процес технічного намагнічування монокристала $\mathrm{Tb}_{0.26} \mathrm{Y}_{2.74} \mathrm{Fe}_{5} \mathrm{O}_{12}$ у температурній області спонтанного орієнтаційного фазового переходу (ОФП). Встановлено, що ОФП розтягнуто у деякому температурному інтервалі, в якому спостерігається співіснування доменів низькотемпературної та високотемпературної магнітних фаз. Виявлено аномалії температурних залежностей коерцитивної сили і магнітооптичної сприйнятливості кристала, зв'язані з перебудовою його доменної структури при спіновій переорієнтації. Інтерпретація отриманих експериментальних результатів виконана в рамках теорії ОФП у кубічному кристалі. Показано, що існуюча теорія адекватно описує еволюцію доменної структури гранату при спонтанній переорієнтації осі легкого намагнічування. 